COMO CITAR ESTE ARTÍCULO:

Serna-Gómez, H.M, Alzate-Acevedo, J., RamírezOspina, D.E. y Castro-Escobar, E.S. (2019). La inserción laboral de los jóvenes en Colombia. Retos y perspectivas. Revista Jurídicas, 16 (1), 42 61. DOI: 10.17151/jurid.2019.16.1.4.

Recibido el 26 de enero de 2018 Aprobado el 7 de noviembre de 2018

\section{La inserción laboral de los jóvenes en Colombia. Retos y perspectivas*}

\author{
Héctor Mauricio Serna-Gómez** \\ Judith Alzate-ACEVedo $* * *$ \\ Duván Emilio Ramírez-OSPINA**** \\ Edisson Stiven Castro-Escobar*****
}

\title{
RESUMEN
}

En este artículo se analiza la inserción laboral de los jóvenes en Colombia, para tal fin se analiza la Gran Encuesta Integrada de Hogares realizada por el Departamento Administrativo Nacional de Estadística - DANE durante el período 2008-2015 para las 13 principales áreas metropolitanas del país. Como principales resultados se encuentra los altos niveles de desempleo de los jóvenes de hogares de bajos ingresos, con bajos niveles de educación y las mujeres jóvenes; adicionalmente, se encontró una alta proporción de jóvenes sin contrato escrito, lo que dificulta el acceso a un trabajo laboral estable y de calidad. Se concluye que es necesario fortalecer las políticas de acceso al trabajo para los jóvenes, dado que persiste el desempleo juvenil y la precariedad laboral.
Palabras clave: mercado laboral, oportunidades de empleo, empleo juvenil, desempleo juvenil.

\footnotetext{
* Este trabajo se deriva del proyecto de investigación "Caracterización del mercado de trabajo en Colombia. Un análisis desde la perspectiva de la segmentación laboral", financiado por la Universidad de Manizales y realizado durante el período febrero de 2015 a marzo de 2017 (código de proyecto en el sistema de investigaciones de la Universidad de Manizales B0601X0402).

* Magíster en economía. Docente Universidad de Manizales, Manizales, Colombia. E-mail: hserna@ umanizales.edu.co. Google Scholar. ORCID: 0000-0002-3945-2843.

*** Magíster en economía, profesional especializado Fundación Luker, Manizales, Colombia. E-mail: judithalzateacevedo@gmail.com. Google Scholar.

ORCID: 0000-0002-7840-9776.

"* Doctor en administración, Docente Universidad de Manizales, Manizales, Colombia. E-mail: merca2@ umanizales.edu.co. Google Scholar ORCID: 0000-0001-5330-9253.

*** Magíster en economía, Docente Universidad de Manizales, Manizales, Colombia. E-mail: ecastro@ umanizales.edu.co. Google Scholar

ORCID: 0000-0002-7798-5050.
} 


\title{
Labor insertion of young people in Colombia. Challenges and perspectives
}

\begin{abstract}
This article analyzes the labor insertion of young people in Colombia. To reach this purpose, the Gran Encuesta Integrada de Hogares (Great Integrated Household Survey) applied by the Departamento Administrativo Nacional de Estadística -DANE(National Administrative Department of Statistics) during the period 2008-2015 to 13 main metropolitan areas in the country was analyzed. The main results are the high levels of unemployment among young people from low-income households, with low levels of education and young women. Also a high proportion of young people working without a written contract was found, which hinders access to stable and quality work. It is concluded that it is necessary to strengthen policies on access to work for young people given that their unemployment and job insecurity persist.
\end{abstract}

KeY wORDs: labor market, employment opportunities, young people employment, young people unemployment. 


\section{Introducción}

En diferentes contextos se ha mencionado la necesidad de generar escenarios de participación de los jóvenes, siendo el más importante el escenario laboral, toda vez que es "la principal fuente de ingreso de las personas, proporciona integridad social y conlleva legitimidad y reconocimiento social. Es también un ámbito de desarrollo interpersonal que facilita los contactos y la integración a redes, y permite la participación en acciones colectivas" (Comisión Económica para América Latina y el Caribe [CEPAL], 2004, p. 205). Los mercados de trabajo son el escenario ideal para que los jóvenes generen procesos de participación ciudadana, en estos se forja el desarrollo interpersonal, se favorece la legitimidad y el reconocimiento como persona.

En dicho sentido, la inserción laboral es entendida como el proceso de incorporación a la actividad económica por parte de los jóvenes, en la cual se da una transición social que viene de la participación en el sistema educativo y estar plenamente vinculado a la familia, hacia la participación en los mercados de trabajo y la generación de independencia familiar (García y Gutiérrez, 1996); por tanto, buenos procesos de inserción favorecen la construcción de ciudadanía por parte de los jóvenes.

Los jóvenes del mundo no están exentos de las problemáticas de inserción laboral, de hecho, todo lo contrario "cada vez es mayor el número de jóvenes que tienen problemas cuando busca trabajo por primera vez, y los niveles de desempleo juvenil son claramente graves en muchos de los Estados miembros de la OIT" (Niall, 2001, p. 9).

Es de destacar que el concepto de juventud varía ampliamente dependiendo el país de procedencia, para la Organización de las Naciones Unidas (ONU) son jóvenes las personas entre los 15 y 24 años (ONU, 1992). Para el caso colombiano se considera una persona joven quien que se encuentre entre los 14 y 28 años (Dirección del Sistema Nacional de Juventud "Colombia Joven", 2013).

Globalmente es posible identificar cuatro aspectos frente al acceso al trabajo en jóvenes: el primero es que luego de la crisis económica internacional del año 2009 se han atenuado los niveles de desempleo juvenil, toda vez que durante el período 2010-2014 la tasa de desempleo de los jóvenes se mantuvo en un $13 \%$, mientras que durante el período 2007-2010 la tasa de desempleo aumentó de un 11,7 \% al $13 \%$ (Organización Internacional del Trabajo [OIT], 2015). El segundo es que bajo el período 2009-2014 el número de jóvenes desempleados se redujo en 3,3 millones desde el pico de la crisis internacional, pasando de 76,6 millones de jóvenes desempleados en 2009 a alrededor de 73,3 millones en 2014 (OIT, 2015). El tercer aspecto para destacar es que para 2014 el 36,7 \% de las personas desocupadas eran jóvenes, mientras que el 2004 eran el 41,5 \% (OIT, 2015). El cuarto aspecto es que a nivel mundial no se ha dado una variación significativa en la relación entre las tasas 
de desempleo de los jóvenes y las tasas de desempleo de los adultos, dado que la diferencia entre ambas tasas durante el período 1995-2014, ha sido tres veces mayor la tasa de los jóvenes frente a la de los adultos, llevando a que en todos los países del mundo las tasas de desempleo juvenil sea mayor que la de los adultos (OIT, 2015).

Lo anterior pone de manifiesto unas condiciones críticas frente a la participación de los jóvenes en los mercados globales de trabajo, toda vez que no se han generado cambios significativos para su participación desde 1995, además establece la necesidad de fortalecer y promulgar nuevos programas gubernamentales que incentiven la vinculación laboral, dado que del total mundial de desocupados para 2014 el 36,7 \% eran jóvenes, y ellos solo representaban una sexta parte de la población mundial para ese mismo año (OIT, 2015).

Al identificar los niveles de desempleo de los jóvenes por regiones del mundo durante el período 2010-2015, se reconoce que en todas las regiones el desempleo es de dos dígitos, siendo mucho más agudos los problemas de participación laboral en África del Norte, donde los niveles de desocupación juvenil alcanzaron tasas de desempleo superior al $28 \%$ promedio durante el período en referencia, luego sigue Oriente Medio con tasas de desempleo del $27,7 \%$ en promedio, mientras que con menores niveles de desempleo se encuentran los países europeos y economías desarrolladas con tasas promedio cercanas al $17 \%$. Para el caso de América Latina las tasas de desempleo juvenil promedio durante el período de análisis fueron del 13,9\%, por debajo de las economías desarrolladas, la razón fue la crisis financiera del 2008 que generó un mayor impacto negativo en dichos países en comparación a los países de América Latina y el Caribe (tabla 1).

Tabla 1. Tasa de desempleo juvenil mundiales y regionales. Período 2010-2015.

\begin{tabular}{lccccccc}
\hline \multicolumn{1}{c}{ REGIONES } & $\mathbf{2 0 1 0}$ & $\mathbf{2 0 1 1}$ & $\mathbf{2 0 1 2}$ & $\mathbf{2 0 1 3}$ & $\mathbf{2 0 1 4 e}$ & $\mathbf{2 0 1 5 p}$ & Promedio \\
\hline MUNDO & 13,0 & 12,9 & 13,0 & 13,0 & 13,0 & 13,1 & 13,0 \\
$\begin{array}{l}\text { Economías desarrolladas y } \\
\text { Unión Europea }\end{array}$ & 18,1 & 17,6 & 18,0 & 17,7 & 16,6 & 16,2 & 17,4 \\
$\begin{array}{l}\text { Europa Central y } \\
\text { Sudoriental }\end{array}$ & 18,9 & 17,9 & 17,4 & 16,8 & 17,2 & 17,6 & 17,6 \\
Asia Oriental & 9,3 & 9,7 & 10,1 & 10,4 & 10,6 & 10,9 & 10,2 \\
Asia Sudoriental y el & 14,7 & 13,1 & 12,7 & 13,5 & 13,6 & 13,6 & 13,5 \\
$\begin{array}{l}\text { Pacífico } \\
\text { Asia Meridional }\end{array}$ & 9,7 & 9,7 & 9,9 & 9,9 & 9,9 & 10,0 & 9,9 \\
América Latina y el Caribe & 15,0 & 14,3 & 13,5 & 13,4 & 13,4 & 13,9 & 13,9 \\
Oriente Medio & 26,1 & 27,6 & 27,6 & 27,9 & 28,2 & 28,7 & 27,7 \\
África del Norte & 24,2 & 27,8 & 29,7 & 30,2 & 30,5 & 30,6 & 28,8 \\
África subsahariana & 12,2 & 12,1 & 12,1 & 11,6 & 11,6 & 11,6 & 11,9 \\
\hline & e = estimado, p proyecciones & & &
\end{tabular}


Según esto, la OIT reconoce que no es fácil ser joven en un mundo bajo los mercados de trabajo actuales, dado que la inestabilidad económica y sus altos grados de incertidumbre "han agravado la crisis de empleo juvenil, dificultando aún más el acceso al empleo para muchos desafortunados jóvenes que buscan trabajo. Y está dificultándolo hasta tal punto que muchos están renunciando a seguir buscándolo" (OIT, 2013, p. 1). De acuerdo con el Banco Interamericano de Desarrollo [BID] (2004), esta fragilidad se debe a que muchos de los jóvenes que intentan ser vinculados al mercado laboral no cuentan con los requerimientos básicos del sector productivo debido a que los sistemas educativos poseen grandes deficiencias, y debido a que la forma en que los jóvenes buscan trabajo es ineficiente, costosa y basada principalmente en métodos informales como contactos personales.

Bajo estas consideraciones, el comprender las posibilidades de acceder a un empleo y las condiciones laborales que hoy día enfrentan los jóvenes en Colombia es fundamental para reconocer cómo ellos participan en la construcción de ciudadanía e identificar los posibles obstáculos que enfrentan para desenvolverse en los contextos actuales. Para el desarrollo del estudio se tomaron los datos de la Gran Encuesta Integrada de Hogares para las 13 principales ciudades del país, esta encuesta es realizada por el Departamento Administrativo Nacional de Estadística -DANE- y bajo sus consideraciones metodológicas presenta capacidad de inferencia para la población objeto de estudio. El período analizado es 20082015, y se analizaron aspectos como: el desempleo juvenil segmentado por estrato social, género, y nivel de educación; la proporción de personas que no estudian y no trabajan por ciudades; condiciones contractuales, proporción de jóvenes que trabajan por cuenta propia nivel de ingresos, entre otros aspectos.

\section{Desafíos para la inserción laboral de los jóvenes}

Como se logró identificar en el apartado anterior, existen grandes debilidades frente a la inserción laboral de los jóvenes en el contexto global, las cuales afectan su bienestar y los procesos de participación ciudadana, pero bajo dichas consideraciones no se debe generalizar que existe un problema de inserción laboral juvenil en los mercados de trabajo, sino que por el contrario los problemas de vinculación al mundo del trabajo se concentran en grupos específicos como lo son jóvenes con bajo nivel de educación, jóvenes de hogares de bajos ingresos, y mujeres jóvenes (Weller, 2006a). Adicionalmente, De la Hoz, Quejada y Yánez (2012) plantean que los jóvenes que presenten un mayor grado de dificultad de acceso a los mercados de trabajo y experimentan períodos de desempleo en sus etapas iniciales de vinculación laboral, serán personas que experimentarán dificultades a futuro para acceder a un empleo y tendrán restricciones en la generación de ingreso, por lo que se convierten en otro de los grupos de atención especial. Bajo estas consideraciones, Weller (2007) plantea que son cuatro los desafíos que deben enfrentar los gobiernos para favorecer la inserción de los jóvenes en el mundo del trabajo, los cuales van en sintonía con lo expuesto por la ONU (1992). 
El primer aspecto que destaca Weller (2007) es aumentar los niveles de empleabilidad, el cual se refiere al conjunto de aptitudes y actitudes que brindan a un individuo la oportunidad de ingresar a un contexto laboral (Campos, 2003). En dicho sentido, Weller (2007) menciona que para favorecer la empleabilidad de los jóvenes en los contextos latinoamericanos se debe fortalecer la educación, buscando que sea pertinente y de mayores niveles de formación; se deben fortalecer las condiciones de intermediación laboral que permitan a los jóvenes identificar las oportunidades de empleo y acceder a puestos de trabajo a partir de procesos de selección meritocráticos.

Frente a esto, la CEPAL (2004) menciona que durante los últimos años los gobiernos de América Latina han promulgado políticas en torno a la cobertura de la educación, con las cuales se ha pretendido impactar de forma positiva la inserción laboral de los jóvenes toda vez que:

(...) la expansión de los sistemas educativos tiene un doble efecto en la oferta laboral juvenil. Primero, un efecto cuantitativo, pues la mayor permanencia de los jóvenes en el sistema reduce la participación laboral; y segundo, un efecto cualitativo, ya que los jóvenes entran al mercado de trabajo con mejores niveles educativos. En consecuencia, en la oferta laboral, una menor presión de participación laboral juvenil y una mayor calidad de la mano de obra de las nuevas cohortes entrantes a los mercados de trabajo tenderían a favorecer la inserción laboral de los jóvenes. (p. 210)

Asimismo, en los últimos años se han creado los servicios públicos de empleo que deben generar una búsqueda efectiva de empleo, capacitar a las personas desocupadas frente a los requerimientos de la empresa y otorgar acceso a servicios sociales a los desempleados mientras encuentran un nuevo empleo (Samaniego, 2002). Tanto la cualificación de los jóvenes como la creación de mecanismos de intermediación entre las empresas y las personas que buscan trabajo deben favorecer las dinámicas de los mercados de trabajo para los jóvenes.

El segundo aspecto que destaca Weller (2007) es favorecer la equidad de género en los mercados de trabajo, en especial para las mujeres jóvenes, toda vez que existen barreras culturales que impiden el acceso de las mujeres a los mercados de trabajo. Para Abramo (2004), si bien es cierto han aumentado los niveles de inserción laboral de las mujeres toda vez que ha disminuido la brecha de participación laboral entre hombres y mujeres y se han aumentado los niveles de ocupación de la mujer, todavía persiste el imaginario de la mujer en los mercados de trabajo como una fuerza de trabajo secundario, originado por tradiciones culturales que han llevado a que todavía persista una división del trabajo bajo género, llevando a que los hombres actúen en el ámbito productivo y las mujeres actúen en el ámbito familiar (Weller, 2007). Estos hechos se ven reflejados incluso en condiciones laborales como el ingreso y los términos de contratación. Por tal motivo "las políticas de 
empleo, específicamente las dirigidas a los jóvenes, deben adoptar un enfoque transversal de género, reconociendo las múltiples trabas y desigualdades que enfrentan las mujeres en el acceso al mercado de trabajo y en el mundo laboral mismo" (Weller, 2007, p. 78).

El tercer aspecto que destaca Weller (2007) es el fortalecer el espíritu emprendedor de los jóvenes, toda vez que quienes pretenden crear empresa y alcanzar una independencia laboral, presentan mayores obstáculos que los adultos que se encuentran en esta misma situación, pero dicha condición no puede ser generalizable y mucho menos la única opción para favorecer la inserción laboral, debido a que no todos los jóvenes tienen como opción crear empresa, no todos tienen conocimiento de los diversos programas que incentivan la creación de empresa (García-García, 2015), y un gran número de ellos con iniciativas como el autoempleo y el microemprendimiento, no logran una inserción laboral adecuada debido a que muchas de estas iniciativas "están ligadas a la supervivencia y la informalidad, de modo que con frecuencia no son una opción de trabajo elegida voluntariamente por los jóvenes, sino más bien una necesidad" (Weller, 2006b, p. 261). Frente a esto Llisterri, Kantis, Angelelli \& Tejerina (2006) realizaron una caracterización del emprendimiento juvenil, encontrando que este es mucho más frecuente en jóvenes de hogares de ingresos bajos que en los de hogares de ingresos medios y altos; esto se origina por la necesidad de buscar mejores oportunidades, por tal motivo Llisterri et al. (2006) proponen que los gobiernos deben incentivar la formación en los jóvenes emprendedores de hogares de bajos ingresos, dado que la opción no debe ser la creación de negocios, sino por el contrario continuar su educación para que de esta manera su vocación por crear empresa se vea fortalecida con conocimientos y habilidades técnicas constituidas en centros de formación técnica y universitaria.

El cuarto aspecto que destaca Weller (2007) es la creación de empleo, toda vez que hoy día los jóvenes perciben un alto grado de dificultad para acceder a buenos puestos de trabajo, debido a las exigencias en cualificación y experiencia laboral que por su edad y juventud en muchos casos es de difícil acceso. Frente a esto se han creado diversos programas como lo son los contratos especiales que permiten favorecer la contratación de los jóvenes, pero con derechos laborales reducidos (en relación con lo salarial, seguridad social y condiciones de despido entre otros), los cuales en muchos casos han favorecido la precariedad laboral juvenil y no la creación de empleos de calidad para los jóvenes.

Siguiendo estas consideraciones, Farné (2009) identifica algunos programas que podrían favorecer la vinculación de los jóvenes en el contexto laboral, entre los más importantes se destacan: el proporcionar servicios de cuidados de infantes en zonas rurales y en zonas periféricas de las ciudades para que las mujeres jóvenes puedan vincularse de forma activa a los mercados de trabajo, el fomentar 
las actividades de emprendimiento en los jóvenes desde la educación secundaria, y el generar una formación pertinente y adecuada para favorecer la vinculación laboral de los jóvenes. Igualmente, Pedraza (2008) destaca la necesidad de crear políticas púbicas que den respuesta a las realidades de los jóvenes, una de las políticas que ha tenido resultados satisfactorios en algunas ciudades del país, es la creación de zonas francas que han fomentado empresas de contact-center con capitales extranjeros, lo que ha permitido la vinculación de jóvenes de bajos recursos al entorno laboral con condiciones dignas de empleo (Castro-Escobar y Serna-Gómez, 2016).

\section{La inserción laboral de los jóvenes colombianos}

Los jóvenes de 14 a 28 años en Colombia presentan dificultad para acceder a condiciones de empleo, prueba de ello son las altas tasas de desempleo juvenil, ésta ha sido persistentemente mayor al nivel de desempleo de las personas mayores a 28 años, Ilegando a tener una diferencia promedio durante el período de estudio de 11 puntos porcentuales (tabla 2).

Tabla 2. Tasa de desempleo en las 13 principales áreas metropolitanas de Colombia de acuerdo con la edad.

\begin{tabular}{llcccccccc}
\hline $\begin{array}{c}\text { Caracterización } \\
\text { etaria }\end{array}$ & \multicolumn{1}{c}{ Tasa } & $\mathbf{2 0 0 8}$ & $\mathbf{2 0 0 9}$ & $\mathbf{2 0 1 0}$ & $\mathbf{2 0 1 1}$ & $\mathbf{2 0 1 2}$ & $\mathbf{2 0 1 3}$ & $\mathbf{2 0 1 4}$ & $\mathbf{2 0 1 5}$ \\
\hline \multirow{2}{*}{ (14 a 28 años) } & Desempleo & 19,8 & 21,6 & 21,1 & 19,6 & 19,0 & 17,7 & 17,1 & 16,3 \\
& TGP* & 56,9 & 59,0 & 59,9 & 61,3 & 62,9 & 62,3 & 63,0 & 62,9 \\
\hline \multirow{2}{*}{ (mayores a 28 años) } & Desempleo & 7,9 & 9,2 & 8,7 & 7,9 & 7,8 & 7,6 & 7,0 & 7,1 \\
& TGP & 70,1 & 72,0 & 73,1 & 73,6 & 74,1 & 73,9 & 74,1 & 74,0 \\
\hline \multirow{2}{*}{$\begin{array}{l}\text { Desempleo 13 áreas } \\
\text { metropolitanas }\end{array}$} & Desempleo & 11,5 & 13,0 & 12,4 & 11,5 & 11,2 & 10,6 & 9,9 & 9,8 \\
& TGP & 65,4 & 67,5 & 68,5 & 69,3 & 70,3 & 70,0 & 70,4 & 70,4 \\
\hline
\end{tabular}

* TGP: Tasa Global de Participación.

Fuente: Elaboración propia con base en datos de la Gran Encuesta Integrada de Hogares realizada por el DANE.

Un aspecto que manifiesta de manera crítica la vinculación de los jóvenes al contexto laboral es la alta proporción de jóvenes desocupados frente al total de desocupados en las 13 principales áreas metropolitanas de Colombia, dado que en promedio durante el período 2008-2015 uno de cada dos desocupados era un joven. Este aspecto resulta ser sumamente crítico por dos razones: la primera es que los jóvenes en las 13 principales ciudades son una cuarta parte de la población ${ }^{1}$, por lo que la alta proporción de desocupados jóvenes frente al total de

${ }^{1}$ Datos generados de la Gran Encuesta Integrada de Hogares realizada por el DANE. 
desocupados, manifiesta una gran ausencia de oportunidades de empleo para la población juvenil; el segundo aspecto es que esta proporción es muy superior a los valores promedios en el contexto global, toda vez que para el año 2014 el 36,7 \% de los desocupados en el mundo eran jóvenes (OIT, 2015), mientras que en las 13 principales ciudades de Colombia esta proporción fue del 51,4% (tabla 3).

Tabla 3. Proporción de jóvenes desocupados frente al total de personas desocupadas en las 13 principales áreas metropolitanas de Colombia.

\begin{tabular}{lcccccccc}
\hline Área & $\mathbf{2 0 0 8}$ & $\mathbf{2 0 0 9}$ & $\mathbf{2 0 1 0}$ & $\mathbf{2 0 1 1}$ & $\mathbf{2 0 1 2}$ & $\mathbf{2 0 1 3}$ & $\mathbf{2 0 1 4}$ & $\mathbf{2 0 1 5}$ \\
\hline $\begin{array}{l}13 \text { principales áreas } \\
\text { metropolitanas de Colombia }\end{array}$ & 53,0 & 50,9 & 51,4 & 52,0 & 51,5 & 50,0 & 51,4 & 49,3 \\
\hline
\end{tabular}

Fuente: Elaboración propia con base en datos de la Gran Encuesta Integrada de Hogares realizada por el DANE.

Al diferenciar los jóvenes de 14 a 28 años y las personas mayores a 28 años de acuerdo con su nivel de educación es posible identificar que tanto la tasa global de participación (que se refiere a la proporción de personas que participan en el mercado laboral) como la tasa de desempleo, difieren de acuerdo con los niveles de educación y a la edad. Concretamente, es posible identificar que para 2008 los jóvenes sin ningún nivel de educación presentaron una mayor tasa de desempleo, toda vez que su tasa es mayor y su nivel de participación en los mercados de trabajo -TGP- es mucho menor, manifestando que la mayoría de jóvenes sin nivel de educación no trabajaban y para quienes deseaban trabajar era difícil acceder a un empleo (tabla 4). Esta condición ha sido persistente en el tiempo pese a que la tasa de desempleo en los jóvenes sin educación pasó de 21,5 en 2008 a 13,6 en 2015, debido a que su tasa global de participación también disminuyó al pasar del $34,6 \%$ en 2008 al 31,1 \% en 2015, aspecto que manifiesta que este descenso en la tasa de desempleo es explicado en gran medida por el aumento en la inactividad en los jóvenes (tabla 4). Un aspecto importante por destacar es que en el último año los jóvenes con título universitario presentan mayores tasas de desempleo que los jóvenes con bajo o sin ningún nivel de educación, lo cual se debe a un mayor nivel de participación de los jóvenes universitarios. 
Tabla 4. Tasa de desempleo juvenil en las 13 principales áreas metropolitanas de Colombia de acuerdo con el nivel de educación.

\begin{tabular}{|c|c|c|c|c|c|c|c|c|c|c|}
\hline $\begin{array}{l}\text { Grupo } \\
\text { etario }\end{array}$ & $\begin{array}{c}\text { Nivel de } \\
\text { educación }\end{array}$ & Tasa & 2008 & 2009 & 2010 & 2011 & 2012 & 2013 & 2014 & 2015 \\
\hline \multirow{10}{*}{$\begin{array}{c}\text { Jóvenes } \\
\text { (14 a } 28 \\
\text { años) }\end{array}$} & \multirow{2}{*}{ Ninguno } & Desempleo & 22,8 & 14,8 & 17,9 & 13,0 & 12,9 & 12,8 & 12,4 & 13,6 \\
\hline & & TGP & 30,1 & 34,4 & 35,2 & 34,1 & 32,1 & 26,1 & 28,1 & 31,1 \\
\hline & \multirow{2}{*}{$\begin{array}{l}\text { Básica } \\
\text { primaria }\end{array}$} & Desempleo & 16,4 & 18,3 & 18,3 & 16,9 & 17,1 & 16,3 & 14,6 & 14,0 \\
\hline & & TGP & 66,7 & 71,9 & 70,1 & 71,0 & 69,8 & 70,9 & 69,6 & 70,2 \\
\hline & \multirow{2}{*}{$\begin{array}{l}\text { Básica } \\
\text { secundaria }\end{array}$} & Desempleo & 20,7 & 22,8 & 21,6 & 20,8 & 19,9 & 17,7 & 18,5 & 17,5 \\
\hline & & TGP & 33,9 & 35,8 & 35,2 & 35,7 & 37,9 & 35,5 & 37,2 & 35,9 \\
\hline & \multirow{2}{*}{ Media } & Desempleo & 21,0 & 22,6 & 22,1 & 20,9 & 19,6 & 18,9 & 17,8 & 16,6 \\
\hline & & TGP & 68,0 & 69,2 & 70,6 & 71,8 & 72,4 & 71,1 & 72,6 & 72,7 \\
\hline & \multirow{2}{*}{$\begin{array}{l}\text { Superior o } \\
\text { universitaria }\end{array}$} & Desempleo & 18,7 & 20,7 & 20,5 & 18,5 & 18,4 & 17,0 & 16,5 & 15,9 \\
\hline & & TGP & 62,9 & 66,4 & 68,2 & 70,3 & 72,4 & 72,2 & 71,6 & 72,1 \\
\hline \multirow{10}{*}{$\begin{array}{c}\text { Mayores } \\
\text { de } 28 \\
\text { años }\end{array}$} & \multirow{2}{*}{ Ninguno } & Desempleo & 9,1 & 9,2 & 9,9 & 8,8 & 8,1 & 7,5 & 7,7 & 7,5 \\
\hline & & TGP & 35,6 & 37,2 & 40,3 & 40,8 & 40,0 & 41,0 & 41,0 & 39,0 \\
\hline & \multirow{2}{*}{$\begin{array}{l}\text { Básica } \\
\text { primaria }\end{array}$} & Desempleo & 7,7 & 9,6 & 8,6 & 8,0 & 7,8 & 7,8 & 6,8 & 6,9 \\
\hline & & TGP & 57,5 & 60,5 & 61,0 & 61,1 & 62,4 & 61,0 & 60,7 & 60,1 \\
\hline & \multirow{2}{*}{$\begin{array}{l}\text { Básica } \\
\text { secundaria }\end{array}$} & Desempleo & 8,7 & 10,4 & 10,0 & 8,1 & 8,5 & 7,7 & 7,6 & 7,3 \\
\hline & & TGP & 70,3 & 72,4 & 73,0 & 73,0 & 73,3 & 72,1 & 72,9 & 72,4 \\
\hline & \multirow{2}{*}{ Media } & Desempleo & 8,4 & 9,5 & 8,9 & 8,3 & 8,0 & 8,0 & 7,3 & 7,2 \\
\hline & & TGP & 75,5 & 78,2 & 79,2 & 79,6 & 80,1 & 79,7 & 79,8 & 80,6 \\
\hline & \multirow{2}{*}{$\begin{array}{l}\text { Superior o } \\
\text { universitaria }\end{array}$} & Desempleo & 6,9 & 7,9 & 7,8 & 7,2 & 7,2 & 7,0 & 6,3 & 6,8 \\
\hline & & TGP & 85,0 & 86,2 & 86,4 & 86,7 & 86,3 & 86,4 & 86,0 & 85,8 \\
\hline
\end{tabular}

Fuente: Elaboración propia con base en datos de la Gran Encuesta Integrada de Hogares realizada por el DANE.

Al analizar los niveles de desempleo de acuerdo con la edad y al sexo es posible identificar que las mujeres jóvenes presentan mayor dificultad para acceder a un empleo, toda vez que ha sido persistente la diferencia cercana a los 6 puntos porcentuales entre los hombres jóvenes y las mujeres jóvenes, adicionalmente la tasa de participación en el TGP de las mujeres jóvenes es mucho menor a la de los hombres jóvenes (tabla 5). Un aspecto a destacar es que existen grandes diferencias en el acceso a los mercados de trabajo entre hombres y mujeres dado que para el caso de las mujeres adultas se encuentra una diferencia persistente en la tasa de desempleo cercana a los 4 puntos porcentuales y una participación en los mercados de trabajo significativamente menor -cercana a los 20 puntos porcentuales en 2015 (tabla 5). 
Tabla 5. Tasa de desempleo juvenil en las 13 principales áreas metropolitanas de Colombia de acuerdo con el sexo.

\begin{tabular}{|c|c|c|c|c|c|c|c|c|c|c|}
\hline $\begin{array}{l}\text { Grupo } \\
\text { etario }\end{array}$ & Sexo & Tasa & 2008 & 2009 & 2010 & 2011 & 2012 & 2013 & 2014 & 2015 \\
\hline \multirow{4}{*}{$\begin{array}{l}\text { Jóvenes } \\
\text { (14 a } 28 \\
\text { años) }\end{array}$} & \multirow{2}{*}{$\begin{array}{l}\text { Hombres } \\
(14 \text { a } 28 \\
\text { años) }\end{array}$} & Desempleo & 17,0 & 18,7 & 18,0 & 16,5 & 16,0 & 14,8 & 14,5 & 13,7 \\
\hline & & TGP & 62,0 & 64,2 & 64,0 & 65,8 & 67,9 & 66,4 & 67,3 & 67,5 \\
\hline & \multirow{2}{*}{$\begin{array}{l}\text { Mujeres } \\
(14 \text { a } 28 \\
\text { años) }\end{array}$} & Desempleo & 23,2 & 24,7 & 24,5 & 23,2 & 22,4 & 20,8 & 20,2 & 19,5 \\
\hline & & TGP & 51,9 & 54,3 & 55,7 & 56,8 & 57,9 & 57,9 & 58,6 & 58,3 \\
\hline \multirow{4}{*}{$\begin{array}{c}\text { Mayores } \\
\text { de } 28 \\
\text { años }\end{array}$} & \multirow{2}{*}{$\begin{array}{l}\text { Hombres } \\
\text { (mayores de } \\
28 \text { años) }\end{array}$} & Desempleo & 7,0 & 8,0 & 7,5 & 6,5 & 6,4 & 6,4 & 5,6 & 5,9 \\
\hline & & TGP & 84,4 & 84,9 & 85,5 & 85,4 & 85,6 & 85,3 & 85,6 & 85,2 \\
\hline & \multirow{2}{*}{$\begin{array}{l}\text { Mujeres } \\
\text { (mayores } 28 \\
\text { años) }\end{array}$} & Desempleo & 9,9 & 10,8 & 10,2 & 9,5 & 9,3 & 9,1 & 8,2 & 8,2 \\
\hline & & TGP & 56,8 & 61,1 & 62,6 & 64,0 & 64,4 & 64,2 & 64,4 & 64,7 \\
\hline
\end{tabular}

Fuente: Elaboración propia con base en datos de la Gran Encuesta Integrada de Hogares realizada por el DANE.

Asimismo al caracterizar los niveles de desempleo de acuerdo con la estratificación del hogar es posible identificar que los jóvenes de bajos estratos (estratos 1 y 2) presentan un mayor nivel de desempleo que los jóvenes de estratos altos (5 y 6), lo cual se debe a las mayores tasas de participación en los mercados de trabajo por la necesidad del hogar de estrato bajo para que sus jóvenes aporten prontamente a los ingresos del hogar (tabla 6).

Tabla 6. Tasa de desempleo juvenil en las 13 principales áreas metropolitanas de Colombia de acuerdo con el estrato donde viven.

\begin{tabular}{|c|c|c|c|c|c|c|c|c|c|}
\hline Grupo etario & Tasa & 2008 & 2009 & 2010 & 2011 & 2012 & 2013 & 2014 & 2015 \\
\hline \multirow{2}{*}{$\begin{array}{l}\text { Jóvenes (14 a } 28 \text { años) } \\
\text { de estratos bajos } \\
\text { (estratos } 1 \text { y } 2)\end{array}$} & Desempleo & 22,3 & 23,1 & 22,3 & 21,2 & 20,1 & 18,8 & 18,4 & 17,1 \\
\hline & TGP & 53,1 & 59,1 & 59,6 & 61,0 & 63,1 & 62,3 & 62,8 & 62,9 \\
\hline \multirow{2}{*}{$\begin{array}{l}\text { Jóvenes (14 a } 28 \text { años) } \\
\text { de estratos medio } \\
\text { (estratos } 3 \text { y } 4)\end{array}$} & Desempleo & 18,0 & 19,9 & 20,0 & 17,9 & 17,8 & 16,6 & 16,0 & 15,2 \\
\hline & TGP & 54,1 & 56,2 & 58,4 & 59,9 & 60,9 & 60,2 & 61,8 & 60,6 \\
\hline \multirow{2}{*}{$\begin{array}{l}\text { Jóvenes (14 a } 28 \\
\text { años) de estratos altos } \\
\text { (estratos } 5 \text { y } 6)\end{array}$} & Desempleo & 10,3 & 11,2 & 14,0 & 12,5 & 13,3 & 12,4 & 12,6 & 10,5 \\
\hline & TGP & 45,1 & 46,6 & 47,5 & 48,2 & 46,2 & 47,9 & 48,4 & 49,9 \\
\hline
\end{tabular}

Fuente: Elaboración propia con base en datos de la Gran Encuesta Integrada de Hogares realizada por el DANE.

Al analizar los niveles de desempleo juvenil en las principales ciudades se puede observar que en todas es superior la tasa de desempleo agregada (tabla 6); condición que es constante tanto para 2008 como para 2015, asimismo se identifican altos niveles de disparidad regional en términos del desempleo, toda vez que para 
2015 se encontraban áreas metropolitanas con una tasa desempleo juvenil por debajo del $15 \%$ y áreas metropolitanas con una tasa de desempleo superior al 20 $\%$. Estas diferencias se hacen evidentes igualmente en las ciudades capitales de regiones históricamente cercanas como lo son el Eje Cafetero -Armenia, Pereira y Manizales-; el Tolima Grande -lbagué y Neiva-; y los Santanderes -Cúcuta y Bucaramanga- (tabla 7).

Tabla 7. Tasa de desempleo juvenil principales áreas metropolitanas. Comparativo 2008-2015.

\begin{tabular}{lcccc}
\hline \multicolumn{1}{c}{$\begin{array}{c}\text { ÁREAS } \\
\text { METROPOLÍTANAS }\end{array}$} & $\begin{array}{c}\text { 2008 } \\
\text { Desempleo } \\
\text { juvenil }\end{array}$ & $\begin{array}{c}\text { Desempleo } \\
\text { (mayores 28 años) }\end{array}$ & $\begin{array}{c}\text { Desempleo } \\
\text { juvenil }\end{array}$ & $\begin{array}{c}\text { Desempleo } \\
\text { (mayores 28 años) }\end{array}$ \\
\hline Cúcuta & 15,1 & 6,3 & 21,5 & 11,6 \\
Ibagué & 30,4 & 14,3 & 21,4 & 8,9 \\
Cali & 19,5 & 8,5 & 19,4 & 8,2 \\
Pereira & 22,7 & 9,7 & 19,5 & 7,9 \\
Medellín & 23,6 & 9,3 & 18,2 & 7,6 \\
Villavicencio & 16,9 & 8,1 & 16,1 & 8,3 \\
Pasto & 24,9 & 9,9 & 18,3 & 7,2 \\
Manizales & 25,0 & 10,3 & 16,9 & 7,0 \\
Bogotá D.C. & 17,3 & 6,8 & 13,7 & 6,6 \\
Cartagena & 23,6 & 7,6 & 17,3 & 5,7 \\
Barranquilla & 21,1 & 6,7 & 17,3 & 5,3 \\
Bucaramanga & 16,2 & 6,4 & 14,1 & 5,3 \\
Montería & 21,4 & 8,6 & 14,1 & 4,9 \\
13 principales áreas & 19,8 & 7,9 & 16,3 & 7,0 \\
metropolitanas & & & &
\end{tabular}

Fuente: Elaboración propia con base en datos de la Gran Encuesta Integrada de Hogares realizada por el DANE.

De otra parte, al analizar las condiciones laborales de los jóvenes de las 13 principales ciudades de Colombia se identifica que durante el período 20082015 el número de jóvenes ocupados con contrato laboral aumentó de forma considerable Ilegando a un 77,7 \% (gráfico 1), esto quiere decir que en 2015, 8 de cada 10 jóvenes que realizaban una actividad laboral tenían un contrato, mientras que para 2008, 5 de cada 10 jóvenes que realizaban una actividad laboral tenían contrato. Pese a este aumento significativo, es importante reconocer que se dio un deterioro en las condiciones contractuales, toda vez que para 2008 el $89 \%$ de los jóvenes que tenían contrato laboral tenían contrato escrito, mientras que para 2015 solo el 65 \% de los jóvenes con contrato laboral tenían contrato 
escrito. Dicha característica genera un alto grado de dificultad para que los jóvenes accedan a beneficios contractuales que por ley están establecidos como: acceso a salud, a pensión, a una administradora de riesgos laborales y al pago de vacaciones y cesantías (gráfico 1).

Gráfico 1. Condiciones contractuales de los jóvenes en las 13 principales áreas metropolitanas de Colombia. Años 2008- 2015.

JÓVENES QUE LABORARON DURANTE EL AÑO 2015

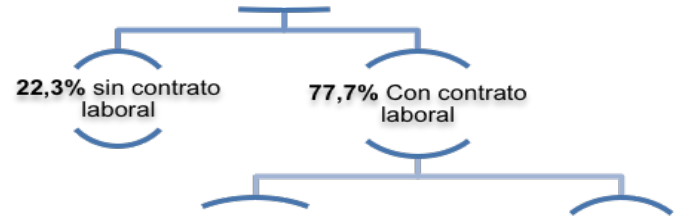

$\mathbf{3 4 , 4} \%$ Contrato verbal

$65,5 \%$ Contrato escrito

$20,2 \%$ de quienes tienen contrato escrito están en
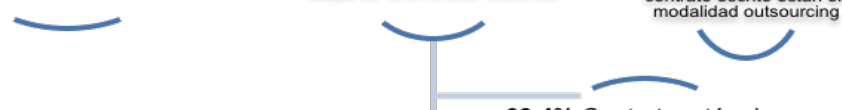

$63,4 \%$ Contrato a término indefinido

$36,6 \%$ Contrato a término

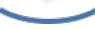

JÓVENES QUE LABORARON DURANTE EL AÑO 2008

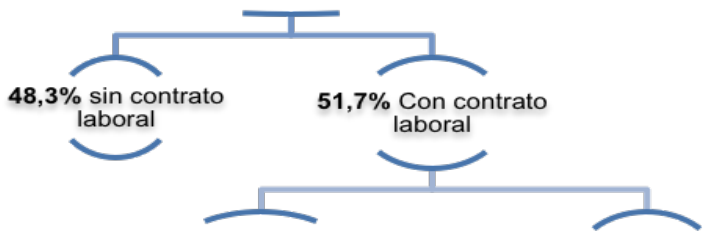

$11 \%$ Contrato verbal

$\mathbf{8 9} \%$ Contrato escrito

$20,2 \%$ de quienes tienen contrato escrito están en modalidad outsourcing
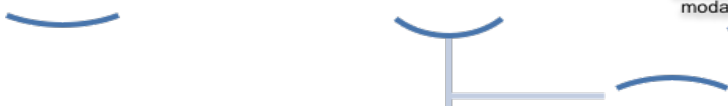

$62,4 \%$ Contrato a término indefinido

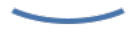

$37,4 \%$ Contrato a término fijo

Fuente: Elaboración propia con base en datos de la Gran Encuesta Integrada de Hogares realizada por el DANE. 
Un aspecto importante a destacar es que del total de jóvenes que se encuentran ocupados realizando alguna actividad, más del 60 \% de ellos consiguió su empleo mediante familiares, amigos o colegas, lo cual lleva a identificar que las relaciones y círculos sociales tanto del joven como de su familia son un factor fundamental para alcanzar una vinculación laboral, toda vez que es el medio más frecuente por los jóvenes de las 13 principales ciudades para acceder a un empleo (tabla 8), asimismo se reconoce la necesidad de continuar fortaleciendo las bolsas y el servicio de empleo, dado que este es un mecanismo de intermediación que favorece la dinámica entre oferentes y demandantes en los mercados de trabajo, no obstante, el bajo uso de este por parte de los jóvenes, manifiesta que todavía falta fortalecer los servicios de empleo (tabla 8).

Tabla 8. Mecanismos para la búsqueda de empleo de los jóvenes ocupados.

\begin{tabular}{lcc}
\hline \multicolumn{1}{c}{ MEDIOS PRINCIPAL PARA ACCEDER A SU EMPLEO ACTUAL } & $\mathbf{2 0 0 8}$ & $\mathbf{2 0 1 5}$ \\
\hline Familiares, amigos o colegas & $68,9 \%$ & $63,9 \%$ \\
Llevando hojas de vida a empresas o empleadores & $16,7 \%$ & $18,5 \%$ \\
Llevando hojas de vida a bolsas de empleo o intermediarios & $5,4 \%$ & $6,1 \%$ \\
Avisos clasificados de empleo & $1,6 \%$ & $3,6 \%$ \\
Por convocatorias & $2,6 \%$ & $3,2 \%$ \\
Por el servicio de empleo SENA & $1,6 \%$ & $2,3 \%$ \\
Otro medio & $3,2 \%$ & $2,4 \%$ \\
TOTAL & $100 \%$ & $100 \%$ \\
\hline
\end{tabular}

Fuente: Elaboración propia con base en datos de la Gran Encuesta Integrada de Hogares realizada por el DANE.

También es importante caracterizar las condiciones salariales y de remuneración de los jóvenes en las 13 principales ciudades de Colombia. Como primer aspecto es de destacar que el $25 \%$ de los jóvenes que trabajan de 40 horas (h) a 48 h no reciben el salario mínimo, porcentaje que ha sido constante desde 2009. Lo segundo para destacar es que el $50 \%$ de los jóvenes que trabajan de 40 h a 48 h en empresas de hasta 5 trabajadores no reciben salario mínimo, por lo que las condiciones mínimas laborales dependerán del nivel de desarrollo y formalización de la empresa donde laboran. El tercer aspecto para destacar es que los ingresos laborales del $1 \%$ de los jóvenes con mejor nivel de remuneración ha tenido una leve reducción durante estos 6 años, teniendo en cuenta que para 2009 ganaban 5 salarios mínimos mensuales legales vigentes (smmlv) y para 2015 ganaban 4,65 smmlv. Por último, el cuarto aspecto a destacar, desde los salarios, es que la formación académica es fundamental para que los jóvenes puedan acceder a un mejor salario, toda vez que se encuentran diferencias salariales entre los jóvenes con formación de básica secundaria y media y los jóvenes con formación universitaria (gráficos 2 y 3). 
Gráfico 2. Niveles de ingresos laborales nominales de los jóvenes que trabajan de 40 a $48 \mathrm{~h}$ semanales de acuerdo con el tamaño de la empresa y su nivel de educación. Año 2015.

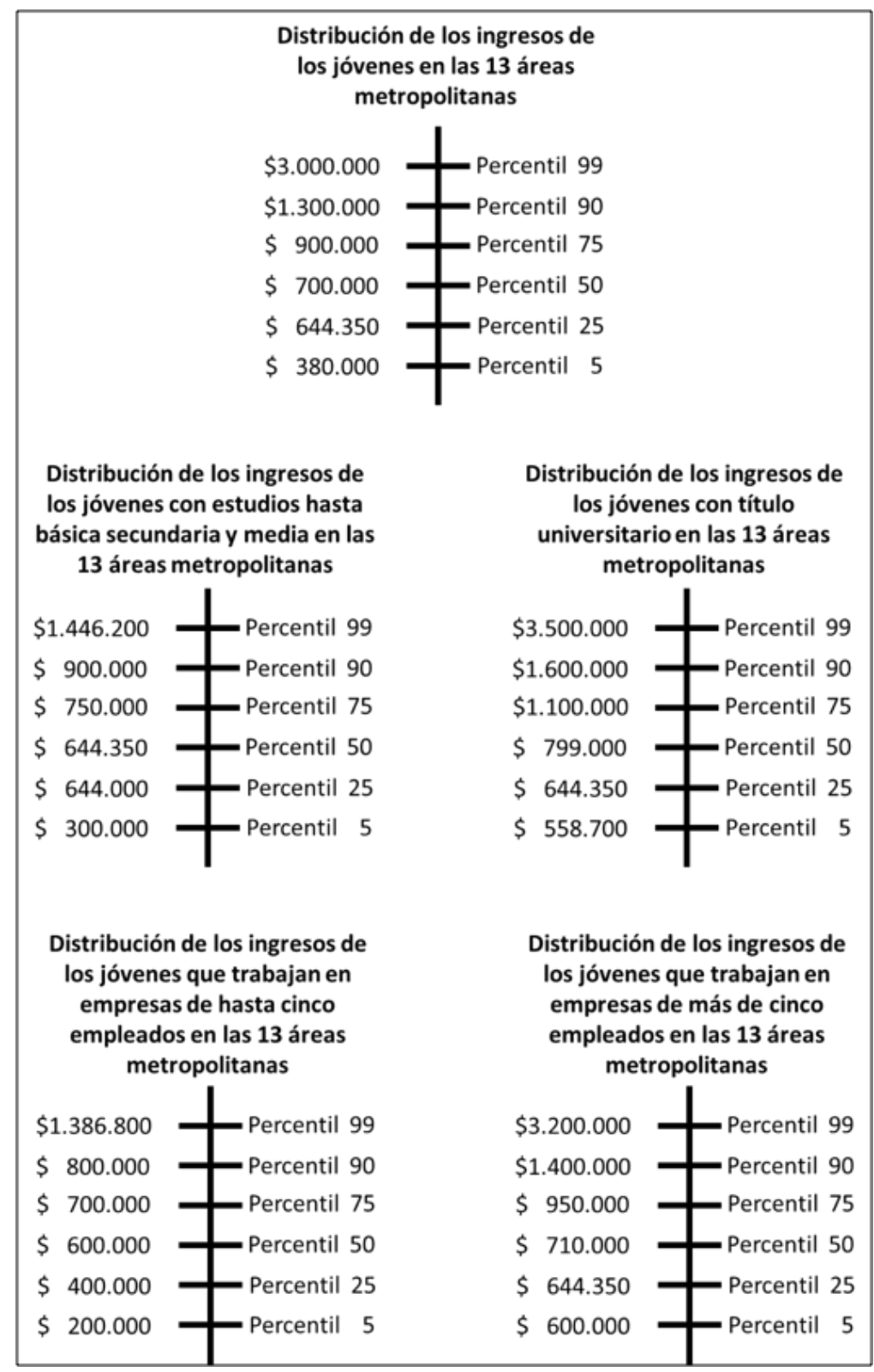

* El salario mínimo en Colombia para el año 2015 fue de \$644.350COP. Fuente: Elaboración propia con base en datos de la Gran Encuesta Integrada de Hogares realizada por el DANE. 
Gráfico 3. Niveles de ingresos laborales nominales de los jóvenes que trabajan de 40 a $48 \mathrm{~h}$ semanales de acuerdo con el tamaño de la empresa y nivel de educación. Año 2009.

\begin{tabular}{|c|c|c|c|}
\hline \multicolumn{4}{|c|}{$\begin{array}{c}\text { Distribución de los ingresos de } \\
\text { los jóvenes en las } 13 \text { áreas } \\
\text { metropolitanas }\end{array}$} \\
\hline & $\$ 2.500 .000$ & rcentil 99 & \\
\hline & $\$ 1.000 .000$ & rcentil 90 & \\
\hline & $\$ 700.000$ & rcentil 75 & \\
\hline & $\$ 500.000$ & rcentil 50 & \\
\hline & $\$ 496.500$ & rcentil 25 & \\
\hline & $\$ 200.000$ & rcentil 5 & \\
\hline $\begin{array}{r}\text { Distribució } \\
\text { los jóvenes } \\
\text { básica secur } \\
13 \text { áreas }\end{array}$ & $\begin{array}{l}\text { ingresos de } \\
\text { udios hasta } \\
\text { media en las } \\
\text { olitanas }\end{array}$ & $\begin{array}{r}\text { Distribuciór } \\
\text { los jóv } \\
\text { universita } \\
\text { met }\end{array}$ & $\begin{array}{l}\text { os ingresos de } \\
\text { con título } \\
\text { las } 13 \text { áreas } \\
\text { litanas }\end{array}$ \\
\hline$\$ 1.200 .000$ & Percentil 99 & $\$ 2.815 .000$ & Percentil 99 \\
\hline$\$ 700.000$ & Percentil 90 & $\$ 1.500 .000$ & - Percentil 90 \\
\hline$\$ 600.000$ & Percentil 75 & $\$ 900.000$ & - Percentil 75 \\
\hline$\$ 497.000$ & Percentil 50 & $\$ 660.000$ & - Percentil 50 \\
\hline$\$ 496.000$ & Percentil 25 & $\$ 498.600$ & - Percentil 25 \\
\hline$\$ 230.000$ & Percentil 5 & $\$ 372.000$ & - Percentil 5 \\
\hline $\begin{array}{r}\text { Distribució } \\
\text { los jóven } \\
\text { empres } \\
\text { emplead } \\
\text { me }\end{array}$ & $\begin{array}{l}\text { ingresos de } \\
\text { abajan en } \\
\text { sta cinco } \\
13 \text { áreas } \\
\text { nas }\end{array}$ & $\begin{array}{r}\text { Distribució } \\
\text { los jóvene } \\
\text { empresa } \\
\text { emplead } \\
\text { met }\end{array}$ & $\begin{array}{l}\text { los ingresos de } \\
\text { e trabajan en } \\
\text { nás de cinco } \\
\text { las } 13 \text { áreas } \\
\text { litanas }\end{array}$ \\
\hline$\$ 1.100 .000$ & Percentil 99 & $\$ 2.500 .000$ & - Percentil 99 \\
\hline$\$ 600.000$ & Percentil 90 & $\$ 1.120 .000$ & - Percentil 90 \\
\hline$\$ 499.500$ & Percentil 75 & $\$ 750.000$ & - Percentil 75 \\
\hline$\$ 400.000$ & Percentil 50 & $\$ 550.000$ & Percentil 50 \\
\hline$\$ 250.000$ & Percentil 25 & $\$ 496.900$ & Percentil 25 \\
\hline$\$ 100.000$ & Percentil 5 & $\$ 360.000$ & - Percentil 5 \\
\hline
\end{tabular}

* El salario mínimo en Colombia para el año 2015 fue de \$496.900COP Fuente: Elaboración propia con base en datos de la Gran Encuesta Integrada de Hogares realizada por el DANE. 
Por último, se destaca la proporción de los jóvenes de 14 a 28 años que trabajan por cuenta propia frente al total de jóvenes ocupados en las 13 principales ciudades. En promedio durante el período 2008-2015, el 25 \% de los jóvenes ocupados trabajaron en actividades por cuenta propia, es decir, decidieron dedicarse a la actividad emprendedora y gestionar su propio negocio (tabla 9) esto lleva a identificar que 1 de cada 4 jóvenes en las 13 principales ciudades del país está realizando una actividad como empresario individual o autónomo, condición que puede ser positiva si efectivamente genera acceso a condiciones de seguridad y estabilidad, pero al analizar las condiciones de acceso a seguridad social de los jóvenes que trabajan por cuenta propia, es posible identificar que para 2015 solo el $14 \%$ de los jóvenes bajo esta condición laboral cotizaban a pensión, y el 82 \% de los jóvenes tenían acceso a seguridad social, de los cuales un poco más del 50 \% tienen acceso a seguridad social mediante régimen subsidiado (tabla 9). Esto pone de manifiesto que si bien es cierto el trabajo por cuenta propia o el ser empresario autónomo se ha convertido en una buena opción para acceder al entorno laboral, todavía presenta condiciones laborales precarias.

Tabla 9. Proporción de jóvenes que trabajan por cuenta propia y son condiciones de acceso a seguridad social en las 13 principales áreas metropolitanas de Colombia.

\begin{tabular}{|c|c|c|c|c|c|c|c|c|}
\hline $\begin{array}{l}\text { Acceso a seguridad } \\
\text { social de los jóvenes } \\
\text { que trabajan por } \\
\text { cuenta propia }\end{array}$ & 2008 & 2009 & 2010 & 2011 & 2012 & 2013 & 2014 & 2015 \\
\hline $\begin{array}{l}\text { Trabajador por } \\
\text { cuenta propia }\end{array}$ & 26,6 & 26,0 & 25,9 & 26,7 & 25,1 & 24,5 & 24,6 & 24,1 \\
\hline $\begin{array}{l}\text { Acceso a seguridad } \\
\text { social (contributivo } \\
\text { o especial) }\end{array}$ & 35,9 & 35,3 & 39,3 & 38,9 & 38,1 & 40,4 & 40,3 & 40,2 \\
\hline $\begin{array}{l}\text { Acceso a seguridad } \\
\text { social (subsidiado) }\end{array}$ & 37,9 & 39,1 & 38,0 & 38,9 & 38,7 & 38,1 & 40,42 & 42,6 \\
\hline Acceso a pensión & 11,2 & 10,8 & 12,7 & 11,7 & 11,5 & 13,2 & 14,8 & 14,1 \\
\hline
\end{tabular}

Fuente: Elaboración propia con base en datos de la Gran Encuesta Integrada de Hogares realizada por el DANE.

Bajo las anteriores consideraciones, el comprender los mercados de trabajo como escenarios de participación de los jóvenes, es fundamental para identificar sus niveles de integración y participación con los sistemas sociales y económicos, "dado que el trabajo se concibe como el eje alrededor del cual gira la condición de miembro de la sociedad" (Benedicto, 2016, p. 927) por lo que discutir frente a sus condiciones laborales es una consideración importante para exponer los actuales retos a los que hoy día se enfrentan los jóvenes en Colombia, el cual es hacer frente a la existencia de mercados de trabajo segmentados y diferenciados de acuerdo con condiciones geográficas y socioeconómicas. 
De acuerdo con esto, ya en los resultados expuestos se reconocen las condiciones que hoy día enfrentan los jóvenes en las 13 principales ciudades de Colombia: primero, una desocupación marcada dado que presentan una tasa de desempleo dos veces superior a la de los adultos y quienes concentran el 50 \% de la población desocupada. Segundo, las bajas tasas de participación laboral, siendo más baja la participación en jóvenes con bajos niveles de educación y en mujeres. Tercero, las contrataciones contractuales en donde aún se persiste por el contrato verbal y en algunos casos la ocupación sin contrato. Cuarto, el autoempleo se convierte en una oportunidad para que los jóvenes puedan acceder a los mercados de trabajo principal, teniendo en cuenta que 1 de cada 4 jóvenes ocupados es emprendedor autónomo, pero se hace evidente que es precaria la inserción laboral bajo esta condición para los ellos, toda vez que solo el $14 \%$ de quienes están bajo estas condiciones laborales tienen acceso a pensión y el 40 \% de ellos tienen acceso a saludo bajo régimen contributivo. Como último aspecto se reconoce que el mecanismo más común por parte de los jóvenes para acceder a un empleo es mediante recomendación de familiares, amigos y conocidos, lo cual genera precarización del trabajo para quienes son de familias de bajos recursos, asimismo pone en evidencia el poco uso por parte del servicio de intermediación laboral ejecutado por los programas del servicio de empleo.

\section{Conclusiones}

La población joven del país evidencia que la transición entre los diferentes niveles de educación y el acceso a fuentes de empleo se convierte en una situación compleja y en muchos casos excluyente; prueba de ello es que para el caso colombiano tener un alto nivel de educación no es un factor que permita mejorar las condiciones de inserción y empleabilidad, esto se identifica con los niveles de desempleo de los jóvenes de acuerdo con su grado de escolaridad, la tasa de desempleo de aquellos con niveles de educación superior o universitaria era superior a la de quienes no tienen nivel de educación, esto se debe por un lado a las altas tasas de participación laboral de quienes tienen formación universitaria, y por el otro al acceso a trabajo informal de aquellos con bajos niveles de educación.

Un segundo aspecto identificado en el estudio es que las mujeres jóvenes presentan mayor dificultad para acceder a un empleo, toda vez que durante el período de análisis ha sido persistente una diferencia promulgada entre los niveles de desempleo entre hombres jóvenes y mujeres jóvenes, adicionalmente la participación laboral de las mujeres jóvenes es mucho menor a la de los hombres jóvenes. Aspecto que pone de manifiesto que para el caso de las mujeres existen condiciones adversas que impiden el acceso a los mercados de trabajo, la cual se agudiza en mayor medida cuando es una mujer joven. Respecto a las condiciones contractuales de los jóvenes de las 13 principales ciudades de Colombia el estudio pudo constatar que el número de ocupados con contrato laboral aumentó de forma considerable 
durante el período 2008-2015, pese a esto se dio un deterioro en las condiciones contractuales, toda vez que durante el período de análisis disminuyó el número de jóvenes con contrato escrito. Dicho aspecto genera barreras para que accedan a beneficios contractuales que por ley se encuentran establecidos.

Un aspecto que se destaca es el alto número de jóvenes que han tomado la decisión de generar recursos propios mediante el autoempleo o el emprendimiento individual o autónomo, esto hace evidente que el emprendimiento es una oportunidad importante para que puedan acceder a los mercados de trabajo, pero se hace evidente la necesidad de fomentar programas que incentiven la calidad del empleo en quienes trabajan por cuenta propia, ya que un gran número no tiene acceso a salud y pensión, para tal fin los programas de emprendimiento deben de generar líneas de acción que incentiven la formalización laboral y promulguen el empleo digno.

Por último, el estudio identificó las condiciones salariales de los jóvenes en las 13 principales ciudades de Colombia, encontrando que el $25 \%$ de quienes trabajan de $40 \mathrm{~h}$ a $48 \mathrm{~h}$ no reciben el salario mínimo, porcentaje que ha sido constante desde 2009. También se encontró que el $50 \%$ de quienes trabajan de 40 h a 48 h en empresas de hasta 5 trabajadores no reciben salario mínimo, por lo que las condiciones mínimas laborales de los jóvenes dependen del grado de formalización de la empresa donde laboran.

Todo lo anterior pone de manifiesto la necesidad de generar políticas nacionales que permitan mejorar las condiciones de empleabilidad de los jóvenes procurando tres aspectos: el primero tiene que ver con el disminuir los niveles de población NINI (población joven que ni estudia ni trabaja), los cuales rondan el $10 \%$, para tal fin se hace necesario generar programas de formación laboral, los cuales procurarán procesos de capacitación asertivos con las necesidades empresariales y favorecerá la vinculación a la actividad laboral. El segundo tiene que ver con generar fuentes de empleo para los jóvenes, toda vez que aún los niveles de desempleo son superiores a los de la población adulta. Para esto se hace necesario evaluar los programas de primer empleo fomentados por el gobierno nacional, esto con el fin de corregir sus debilidades y mejorarlo desde sus fortalezas. Por último, se deben mejorar los niveles de formalización laboral, puesto que el 50 \% de los jóvenes que trabajan en empresas de hasta 5 empleados no tienen contrato laboral, en este caso particular se deben incentivar los contratos de ley mediante programas de desarrollo empresarial, buscando que los establecimientos informales accedan a recursos de capitalización desde el cumplimiento de sus obligaciones contractuales. 


\section{Referencias bibliográficas}

Abramo, L. (2004). ¿Inserción laboral de las mujeres en América Latina: una fuerza de trabajo secundaria? Estudios feministas, 12(2), 224-235.

Banco Interamericano de Desarrollo (BID). (2004). Good jobs Wanted. Labor Markets in Latin America. Economic and Social Progress Report.Washington, D.C.: Banco Interamericano de Desarollo.

Benedicto, J. (2016). La ciudadanía juvenil: Un enfoque basado en las experiencias vitales de los jóvenes. Revista Latinoamericana de Ciencias Sociales, Niñez y Juventud, 14(2), 925-938.

Campos, G. (2003). Implicaciones económicas del concepto de empleabilidad. Aportes, 8(23), 101 111.

Castro-Escobar, E.S. y Serna-Gómez, H.M. (2016). Calidad del empleo en Organizaciones de Servicios de Contact-Center en Manizales, Colombia. Revista Latinoamericana de Ciencias Sociales, Niñez y Juventud, 14(1), 205- 219.

Comisión Económica para América Latina y el Caribe (CEPAL). (2004). La juventud en Iberoamérica. Tendencias y urgencias. Santiago, Chile: CEPAL.

De la Hoz, F.J., Quejada, R. y Yánez, M. (2012). El desempleo juvenil: problema de efectos perpetuos. Revista Latinoamericana de Ciencias Sociales, Niñez y Juventud, 10(1), 427-439.

Dirección del Sistema Nacional de Juventud "Colombia Joven". (2013). Ley estatutaria 1622 de 2013. Estatuto de Ciudadanía Juvenil. Bogotá, Colombia: Dirección del Sistema Nacional de Juventud.

Farné, S. (2009). Políticas para la inserción laboral de mujeres y jóvenes en Colombia. Santiago de Chile: CEPAL.

García, J.M. y Gutiérrez, R. (1996). Inserción laboral y desigualdad en el mercado de trabajo: Cuestiones teóricas. Revista Española de Investigaciones Sociológicas, 75, 269-293.

García-García, V.D. (2015) Emprendimiento empresarial juvenil: Una evaluación con jóvenes estudiantes de universidad. Revista Latinoamericana de Ciencias Sociales, Niñez y Juventud, 13(2),1221-1236.

Llisterri, J.J., Kantis, H., Angelilli, P. \& Tejerina, L. (2006). Is youth entrepreneurship a necessity or an opportunity? A first exploration of household and new enterprise surveys in Latin America. Washington: Inter-American Development Bank.

Niall, O.H. (2001). Desempleo juvenil y política de empleo. Madrid: Ministerio del Trabajo y Asuntos Sociales.

Organización Internacional del Trabajo (OIT). (2013). Tendencias mundiales del empleo juvenil. Una generación en peligro. Ginebra: OIT.

Organización Internacional del Trabajo (OIT). (2015). Tendencias mundiales del empleo juvenil. Promover la inversión en empleos decentes para los jóvenes. Ginebra: OIT.

Organización de las Naciones Unidas (ONU). (1992). Statistical charts and indicators on the situation of youth, 1970 - 1990. New York: ONU.

Pedraza, A.C. (2008). El mercado laboral de los jóvenes y las jóvenes de Colombia: realidades y respuestas políticas actuales. Revista Latinoamericana de Ciencias Sociales, Niñez y Juventud, 6(2), 853-884.

Samaniego, N. (2002). Las políticas de mercado de trabajo y su evaluación en América Latina. Serie 19 macroeconomía del desarrollo. Santiago, Chile: CEPAL.

Weller, J. (2006a). Problemas de la inserción laboral de la población juvenil en América Latina. Papeles de población. 12(49), 9-36.

Weller, J. (2006b). Los jóvenes y el empleo en América Latina. Desafíos y perspectivas ante el nuevo escenario laboral. Bogotá, Colombia: CEPAL.

Weller, J. (2007). La inserción laboral de los jóvenes: características, tensiones y desafíos. Revista de la CEPAL. (92), 61-82. 